\title{
The linear-dependence of adhesion strength and adhesion range on temperature in soft membranes
}

\author{
Zhenghua Chang ${ }^{\mathrm{a}, \mathrm{c}}$, Ronggui Yang ${ }^{\mathrm{b}, *}$, Yujie Wei ${ }^{\mathrm{a}, \mathrm{c}, *}$ \\ ${ }^{a}$ LNM, Institute of Mechanics, Chinese Academy of Sciences, Beijing 100190, People's Republic of China \\ b School of Energy and Power Engineering, Huazhong University of Science and Technology, Wuhan, Hubei, 430074, China \\ ' School of Engineering Sciences, University of Chinese Academy of Sciences, Beijing 100049, People's Republic of China
}

\section{A R T I C L E I N F O}

\section{Article history:}

Received 12 June 2019

Revised 1 August 2019

Accepted 16 August 2019

Available online 20 August 2019

\section{Keywords:}

Soft membrane

Adhesion

Ripples

Thermal undulation

Helfrich Hamiltonian

\begin{abstract}
A B S T R A C T
The adhesion of a soft membrane plays a central role for the functionality of many biological and engineering systems, from cell adhesion to advanced materials. In this work, we demonstrated via a combination of statistical mechanics analysis and molecular dynamics (MD) simulations that the adhesion of a membrane-buffered interface could be significantly modulated due to thermally induced surface corrugations in the membrane. Not expected from classical mechanics theories, the adhesion strength is highly temperaturesensitive and reduces linearly with increasing temperature. Through statistical mechanics analysis, the dependence of adhesion on temperature is found to result from thermally induced ripples which facilitate long-range adhesion between the soft membrane like graphene sheets or lipid bilayers with the substrate. The results from MD simulations agree well with the statistical mechanics analysis. Such surprising findings may pave the way to understand temperature-sensitive kinetics in many biological systems where the interaction of cell membranes with extra-cellular environments is pivotal.
\end{abstract}

(c) 2019 Elsevier Ltd. All rights reserved.

\section{Introduction}

The interaction between a solid and a substrate depends heavily on both of their rigidities. Interface adhesion becomes important when either side of the interacting pair becomes soft. The significance of such mechanical behavior is manifested in many biological systems (Seifert and Lipowsky, 1990; Swain and Andelman, 1999; Fenz et al., 2017) and engineering applications (Drotlef et al., 2017; Yang and Suo, 2018; Yu and Cheng, 2018). When a soft matter is at the limit of one-atom thin membrane, the bonding between the two interacting surfaces may experience a transition from the deterministic interaction to statistical nature. The interface corrugates due to the formation of thermally induced ripples in the membrane as exemplified in graphene, which gives rise to spatial and temporal variation of bonding sites (Deng and Berry, 2016). In practical applications that involve soft membranes and substrate systems (Castellana and Cremer, 2006; Zhao et al., 2017), lattice mismatch (Deng et al., 2017; Gao et al., 2010), surface roughness of the substrates (Ishighmi et al., 2007; Xing and Faller, 2008; Geringer et al., 2009), the mismatch of thermal expansion coefficients (Deng et al., 2017), chemical environment (Dobereiner et al., 1999; Fenz et al., 2017), local composition of the membrane (Devaux, 1991; Dobereiner, 1999), pre-straining (Zang et al., 2013), and pre-existing defects (Seung and Nelson, 1988; Shenoy et al., 2008; Wei et al., 2012; Wei and Yang, 2019) can all induce surface corrugations.

\footnotetext{
* Corresponding authors.

E-mail addresses: ronggui@hust.edu.cn (R. Yang), yujie_wei@lnm.imech.ac.cn (Y. Wei).
} 
The interface interaction of corrugated membranes, which is essential for the cellular signal transduction and functioning of flexible devices, has attracted considerable attention (Katsaras and Gutberlet, 2000; Li and Song, 2018). Other than the effect of direct physical force (van der Waals force, Coulomb force or hydration force, etc.), surface undulations also influence the interaction between the membrane and the neighboring media (Helfrich, 1978). Substantial progress has been achieved in the understanding and prediction of such interface interaction. As originally proposed by Helfrich (1978), surface corrugation would be suppressed if the soft membranes approach each other and the undulation force is induced. His analysis showed that the undulation force $(F)$ is in reverse proportion to the cubic of the average separation $(a)$ with the counterpart of the system. However, inconsistence between experiments for biological lipid bilayers (Petrache et al., 1998) and Helfrich's theory shows that the hard confinement theory underestimates the force. Through Fourier Monte Carlo simulation, Gouliaev and Nagle (1998) proposed a method for the force calculation of soft confinements. Theoretical analysis carried out by Podgornik and Parsegian (1992) proposed an $F$ versus $a$ relationship in the exponential functional form to describe the decay of the undulation force when the membranes separates. Through statistic mechanics analysis, Freund proposed another power law form of $F-a$ relationship that the undulation force varies inversely with the separation of two soft membranes (Freund, 2013). Later on Li and Song (2016) unified the theory of Helfrich (1978) and Freund (2013) and found that the exponent of both power laws is determined by the wave number of surface corrugation.

The statistic mechanics based Helfrich model (Helfrich, 1978) and Freund model (Freund, 2013) represent a significant step to shed light on the interaction between soft membranes with surface undulations, in particular for those ultra-thin ones like single-layer graphene and lipid bilayers. The models are capable of depicting the repulsion induced by surface corrugations when the membranes approach each other. When placed further away from their initial equilibrium gap, two soft membranes could be attractive. Such adhesive behavior can of course be influenced by the morphology of the mutual surface. Temperature then plays a key role as it governs the surface roughening of thin membranes. As a result, it naturally raises the interest in the role of temperature-governed thermal fluctuation on adhesion. Indeed, even the rigidities of soft membranes (Helfrich, 1978; Wei et al., 2013) could be influenced by temperature (Zelisko et al., 2017). The central idea of this work is to demonstrate how temperature induced surface morphology may alter the adhesion of ultra-thin membranes serving as a buffer layer or on substrates.

To demonstrate the statistical nature of the thermally induced ripples and the implication to the mechanical behavior of the membrane-buffered interface, we first adopted the well-studied single-layer graphene (SLG) as the buffer layer. The importance of ultra-low bending rigidity of SLGs has been recognized when studying the interaction of graphene with a nanoscale scratching, friction (Lee et al., 2010), or graphene wrapping of cells (Li et al., 2013; Wang et al., 2013). In particular, the bending rigidity of SLG was found to be $\sim 1.44 \mathrm{eV}$ (Wei et al., 2013). Using SLG as the model membrane material, we focused on understanding the impact of the thermally induced rippling in SLG on the interface adhesion, to establish a relationship between temperature and the interaction of SLG with the substrate, and to demonstrate enhanced adhesion range due to the thermally induced ripples. The lipid bilayer of cells, a biological membrane that is of broad interest with the bending rigidity very close to that of graphene (Lipowsky, 1991), were further studied to quantitatively confirm the extension of the adhesion range.

\section{Analysis on surface corrugations}

\subsection{Molecular dynamics simulations}

The morphology of membranes can be influenced by thermal fluctuation and such a behavior differs greatly from that of bulk materials. We performed a series of molecular dynamics (MD) simulations to explore the spontaneous thermallyinduced ripples of a single layer graphene supported by a copper substrate. The MD simulations regarding the graphenesubstrate system were performed using LAMMPS package (Plimpton, 1995) and visualized through Ovito (Stukowski, 2010). The graphene sheet residing on a $\mathrm{Cu}$ (111) surface has a dimension of $498 \mathrm{~nm} \times 511 \mathrm{~nm}$, which is sufficiently large to allow us to extract the statistical information of rippling. Periodic boundary conditions were applied in the plane (both $\mathrm{x}$ and $\mathrm{y}$ directions) to the system. To focus on the morphology of a single layer graphene as it interacts with a substrate at different temperatures, we constrained the $\mathrm{Cu}$ atoms in order to avoid the effect of substrate surface roughness on the ripples of graphene sheets.

The AIREBO (Stuart et al., 2000) potential was employed to describe C-C interaction, and the EAM (Foiles et al., 1986) potential for $\mathrm{Cu}-\mathrm{Cu}$ interactions. The van der Waals interaction between the graphene membrane and the copper substrate was presented by Lennard-Jones (L-J) pair potential

$$
U(r)=4 \alpha \varepsilon\left[\left(\frac{r_{0}}{r}\right)^{12}-\left(\frac{r_{0}}{r}\right)^{6}\right],
$$

where $\varepsilon=0.0168 \mathrm{eV}, r_{0}=2.2 \AA$ (Xu and Buehler, 2010; Shi et al., 2012) and $r$ stands for the interatomic distance, respectively. Here we introduce a dimensionless parameter $\alpha$, and $\alpha \varepsilon$ is now the potential well depth, or more specifically, the interaction strength among $\mathrm{C}$ and $\mathrm{Cu}$ atoms. For $\alpha=0$, the graphene is free-standing and $\alpha=1$ stands for the standard L-J interaction between $\mathrm{C}-\mathrm{Cu}$.

The system was equilibrated at finite temperatures $(100 \mathrm{~K} \sim 1200 \mathrm{~K})$ in NVT (fixed atom number, sample volume, and temperature) ensembles for 200,000 time steps, and each time step is assumed to be $0.5 f$ s. The interfacial interacting 


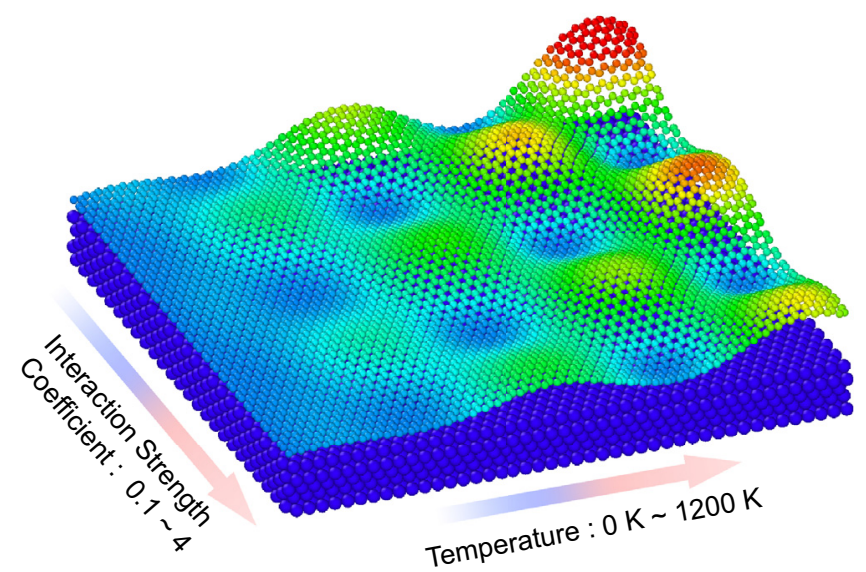

Fig. 1. A sketch to demonstrate surface morphology of an ultra-soft membrane on substrate, where surface rippling is governed not only by the interaction strength (normalized) of membrane atoms and molecules with substrate, but also temperature.
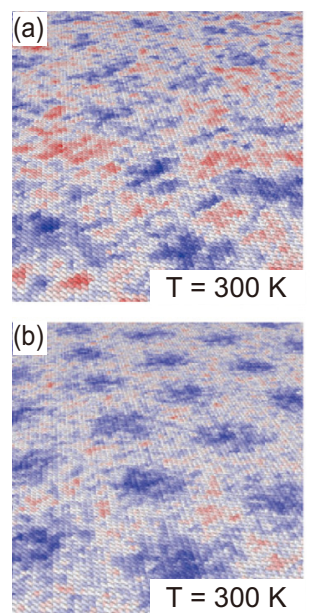

(c)

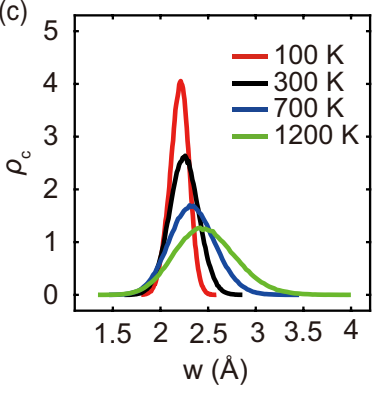

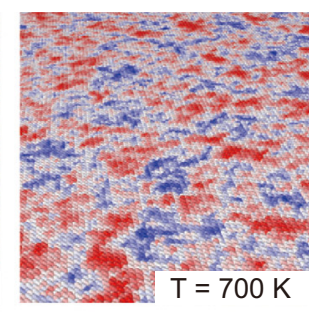
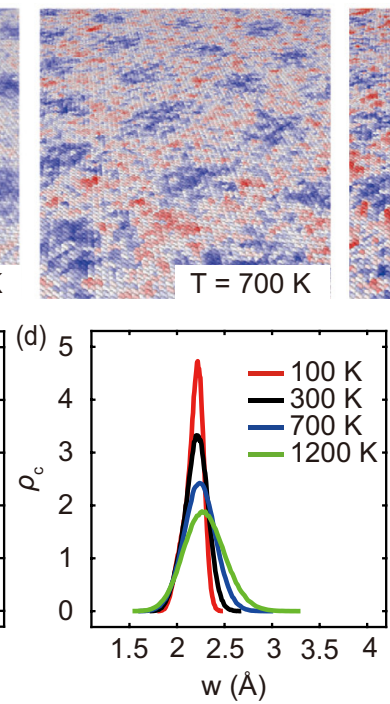

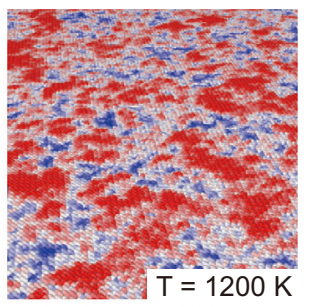

$3.92 \AA$
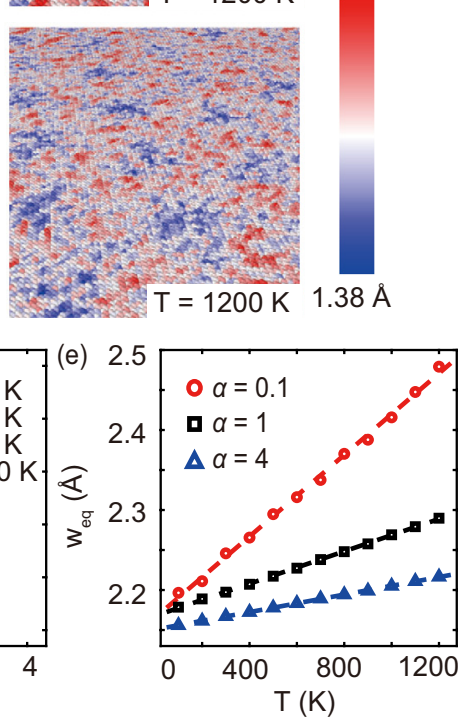

Fig. 2. The morphology of a SLG after relaxation. (a) and (b), The height contour of a SLG relaxed at $300 \mathrm{~K}, 700 \mathrm{~K}$ and $1200 \mathrm{~K}$ on a copper substrate assuming different interaction strength $\alpha=0.1$ and 1, respectively. (c) and (d), The probability distribution $\rho_{c}(w)$ of the normal distance $w$ of carbon atoms above the copper substrate with $\alpha=0.1$ and 1 at different temperatures, respectively. (e) The average separations between the SLG and the substrate of $\alpha=0.1,1$ and 4 at various temperatures.

energy reached a stable value (with a relative variation below $0.003 \%$ ) after the first $10 \mathrm{ps.} \mathrm{The} \mathrm{relatively} \mathrm{long} \mathrm{equilibration}$ would ensure reliable statistical information.

Thermal undulation influences dramatically the morphology of SLG and the influence can be modulated by temperature and the substrate, as straightforwardly seen in Fig. 1. Fig. 2 shows the morphology of SLG after relaxation using MD simulations with the NVT ensemble in detail. Corrugation height of SLG increases with the increase of temperature or the decrease of the bonding strength to the substrate, as seen in Fig. 2(a) and (b). The probability distribution $\rho_{c}$ of the normal distance $w$ between carbon atoms in the graphene and the $\mathrm{Cu}$ (111) plane as a function of interaction strength $\alpha=0.1$ and 1 at different temperatures with $T=300 \mathrm{~K}, 700 \mathrm{~K}$ to $1200 \mathrm{~K}$ are shown in Fig. 2(c) and (d), which can be fit to the Gaussian 
(a)

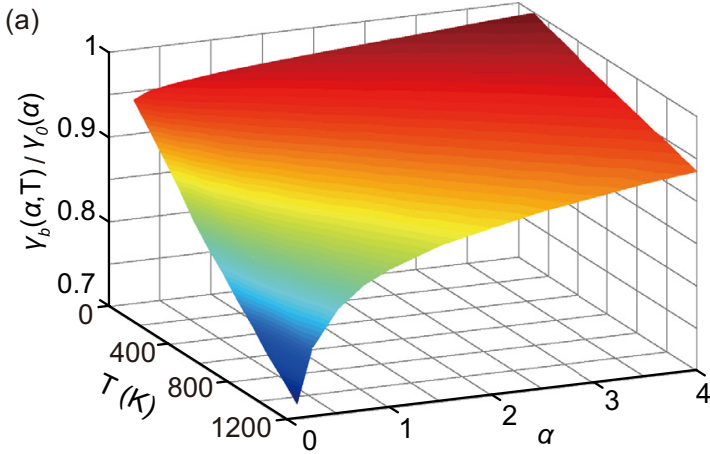

(b)

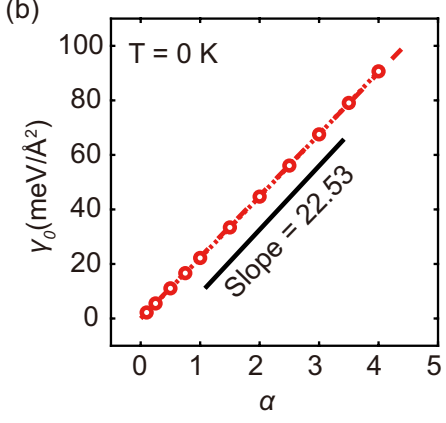

Fig. 3. The temperature dependence of binding energy of a graphene membrane on a Cu substrate under thermal undulation. (a) The binding energy $\gamma_{b}(T$, $\alpha$ ) at a given temperature T and adhesion energy $\alpha$ was normalized by the corresponding binding energy $\gamma_{0}(\alpha)$ of a flat graphene membrane on the same substrate. (b) The linear dependence of $\gamma_{0}(\alpha)$ on $\alpha$.

distribution in the form of

$$
\rho_{c}(w)=\frac{1}{\sqrt{2 \pi\left\langle D_{w}\right\rangle}} e^{-\frac{\left(w-w_{e}\right)^{2}}{2\left(D_{w}\right\rangle}},
$$

with $\left\langle D_{w}\right\rangle$ being the variance of the distribution. The average separation $w_{e q}$, i.e. the normal distance at equilibrium, between SLG and the substrate of $\alpha=0.1,1$ and 4 at various temperatures are more clearly shown in Fig. 2(e). There are several interesting observations regarding to the normal distances of carbon atoms in the graphene sheet residing on the $\mathrm{Cu}$ (111) plane: (1) the distance follows a Gaussian distribution within the interaction strength and the temperature range we explored. (2) Both the mean distance and its standard deviation, which physically represent the amplitude and the size of thermally-induced ripples, increase with the increase of temperature or the decrease of the interaction strength. The Moiré patterns which were well documented in literature (Deng et al., 2017; Gao et al., 2010) are observed when the C-Cu interaction is strong $(\alpha=4)$ and the temperature is low (left side of Fig. 2(b)). The periodicity of the Moire patterns may vary with the lattice constants of graphene membrane and the substrate, as well as the relative orientation of the graphene with the atomic plane of the substrate.

The phenomena shown in Fig. 2 may be more straightforwardly interpreted by showing the average binding energy $\gamma_{b}$ as a function of temperature and the $\mathrm{C}-\mathrm{Cu}$ interaction strength. An area-weighted average energy between the SLG and the copper substrate is obtained by dividing the total interaction with $N S_{0}$, where $N$ is the total number of carbon atoms in the simulation system and $S_{0}=\frac{3 \sqrt{3} d^{2}}{4}=2.63 \AA^{2}$ is the planar footprint of a carbon atom in graphene with $d$ being the C-C bond length. The binding energy $\gamma_{b}(T, \alpha)$ is normalized by $\gamma_{0}(\alpha)$ which is the binding energy when the SLG is flat (see Fig. 3(a)). $\gamma_{0}(\alpha)$ has a negligible dependence on temperature and is proportional to the adhesion energy, $\gamma_{0}(\alpha)=22.53 \alpha \mathrm{meV}$, as shown in Fig. 3(b). The binding energy becomes weaker at higher temperatures or when the C-Cu interaction is weaker, indicating a larger separation between the SLG and the substrate.

\subsection{Statistical mechanics analysis}

The statistical mechanics analysis on thermally induced ripples is articulated below (Nelson and Peliti, 1987; Fasolino et al., 2007; Gao and Huang, 2014; Wang et al., 2016; Ahmadpoor et al., 2017). For a rectangular SLG containing $N$ carbon atoms, we may consider it as a continuous thin membrane with an area of $N S_{0}$ when $\sqrt{N} \gg 1$. Let the in-plane position vector of a carbon atom be $\boldsymbol{r}=(x, y)$ and its normal distance to the substrate be $w(\boldsymbol{r})$. We adopt the canonical distribution to establish the relationship among $w, T$ and $\alpha$. For the Hamiltonian $H$ which is a function of $w$, we may obtain its probability density function $\rho$ in the form of (Weiner, 2002)

$$
\rho=\frac{1}{Z} \exp \left(\frac{-H}{k T}\right),
$$

where the partition function $Z$ is written as

$$
Z=\int \exp \left(\frac{-H}{k T}\right),
$$

and $k$ is the Boltzmann constant. Without loss of generality, the contribution of $H$ comes from two parts: the surface energy of $\mathrm{C}-\mathrm{Cu}$ interaction $\left(E_{C_{-}-\mathrm{u}}\right)$ and the configuration energy within the graphene sheet $\left(E_{C_{-}-}\right)$. The surface energy $E_{C-C u}$ is a summation of the interaction between all $\mathrm{C}-\mathrm{Cu}$ pairs, which can be approximated as

$$
E_{C-C u}=-\alpha \varepsilon N+\int_{N S_{0}} \frac{\alpha \beta}{2}\left(w-w_{e q}\right)^{2} d S,
$$


where $\beta$ is the coefficient of the second-order term of the Taylor series of the L-J potential, $\beta=\frac{72 \varepsilon}{w_{e q}^{2} S_{0}}$ and has a unit of $e V / \AA^{4}$. At high temperature, ripples can form and the corresponding configurational energy can be described by the Helfrich Hamiltonian (Helfrich, 1973; Lipowsky, 1991)

$$
E_{C-C}=\int_{N S_{0}}\left[\frac{1}{2} \kappa_{B}\left(C_{1}+C_{2}\right)^{2}+\kappa_{G} C_{1} C_{2}\right] d S
$$

where $C_{1}$ and $C_{2}$ refer to the two principal curvatures of a ripple. $\kappa_{B}$ and $\kappa_{G}$ are the bending rigidity and the Gaussian bending stiffness of SLG where typical values may be found in the work of Wei et al. (2013). According to Gauss-Bonnet theorem, if the topology of a membrane with no boundaries remains fixed, the Gaussian curvature becomes a constant and the second term in Eq. (4b) is neglected (Nelson et al., 2004; Abbena et al., 2006). We hence have

$$
H=E_{C-C u}+E_{C-C}=-\alpha \varepsilon N+\int_{N S_{0}} \frac{\alpha \beta}{2}\left(w-w_{e q}\right)^{2} d S+\int_{N S_{0}} \frac{1}{2} \kappa_{B}\left(\nabla^{2} w\right)^{2} d S .
$$

Owing to the periodic boundary condition, the Fourier series of $w(\boldsymbol{r})$ can be rewritten as

$$
w(\boldsymbol{r})=\bar{w}(0)+\sum_{q_{x}=\frac{2 n_{x} \pi}{\sqrt{N S_{0}}}, q_{y}=\frac{2 n_{y} \pi}{\sqrt{N S_{0}}}} \bar{w}(\boldsymbol{q}) e^{-i \boldsymbol{q} \cdot \boldsymbol{r}},
$$

with $\boldsymbol{q}=\left(q_{x}, q_{y}\right)$ being the wave numbers and $n_{x}$ and $n_{y}$ are positive integers. Since the wave length should be within an interval from atomic distance $d$ to the length of the graphene sheet $\sqrt{N S_{0}}$, we have $|\boldsymbol{q}| \in\left(\frac{2 \pi}{\sqrt{N S_{0}}}, \frac{2 \pi}{d}\right)$. The coefficients in Eq. (5a) are determined to be

$$
\bar{w}(\boldsymbol{q})=\frac{1}{N S_{0}} \int_{N S_{0}} w(\boldsymbol{r}) e^{-i \boldsymbol{q} \cdot \boldsymbol{r}} d \boldsymbol{r} .
$$

Specifically, $\bar{w}(0)$ in Eq. (5a) represents the average separation between the graphene and the substrate, hence $\bar{w}(0)=$ $w_{\text {eq. }}$. Combining Eqs. (3b), (4c) and (5a), we obtain the partition function in the form of

$$
\mathrm{Z}=e^{\frac{\alpha \varepsilon N}{k T}} \prod_{\boldsymbol{q}} \sqrt{\frac{2 \pi k T}{N S_{0}\left(\kappa_{B}|\boldsymbol{q}|^{4}+\alpha \beta\right)}},
$$

Now taking the magnitude of $\bar{w}(\boldsymbol{q})$ as a statistic parameter, which follows the distribution given in Eq. (3a), the square average of amplitudes for a certain $\boldsymbol{q}$ is given as

$$
\left\langle|\bar{w}(\boldsymbol{q})|^{2}\right\rangle=\frac{1}{Z} \int_{-\infty}^{\infty} \cdots \int_{-\infty}^{\infty}|\bar{w}(\boldsymbol{q})|^{2} \exp \left(-\frac{H}{k T}\right) \prod_{\boldsymbol{q}} d|\bar{w}(\boldsymbol{q})|=\frac{k T}{N S_{0}\left(\kappa_{B}|\boldsymbol{q}|^{4}+\alpha \beta\right)},
$$

For each configuration, the variance of the height distribution is defined as $D_{w}$, which is written as

$$
D_{w}=\frac{1}{N S_{0}} \int_{N S_{0}}\left[w(\boldsymbol{r})-w_{e q}\right]^{2} d \boldsymbol{r}
$$

With Eq. (5a) and noting $\bar{w}(0)=w_{\text {eq }}$, we obtain

$$
D_{w}=\sum_{\boldsymbol{q}}|\bar{w}(\boldsymbol{q})|^{2} .
$$

Thus, the average $D_{w}$ for all possible configurations becomes

$$
\left\langle D_{w}\right\rangle=\sum_{q}\left\langle|\bar{w}(\boldsymbol{q})|^{2}\right\rangle=\sum_{\boldsymbol{q}} \frac{k T}{N S_{0}\left(\kappa_{\boldsymbol{B}}|\boldsymbol{q}|^{4}+\alpha \beta\right)} .
$$

Eq. (7d) gives the variance of the height distribution of carbon atoms. As $\sqrt{N S_{0}} \gg 2 \pi$, we can convert the summation form of Eq. (7d) to an integral with $d q_{x}=d q_{y}=\frac{2 \pi}{\sqrt{N S_{0}}}$,

$$
\left\langle D_{w}\right\rangle=\iint \frac{k T}{N S_{0}\left(\kappa_{B}|\boldsymbol{q}|^{4}+\alpha \beta\right)}\left(\frac{\sqrt{N S_{0}}}{2 \pi}\right)^{2} d q_{x} d q_{y},
$$

which could be further written in the polar coordinate as

$$
\left\langle D_{w}\right\rangle=\left(\frac{\sqrt{N S_{0}}}{2 \pi}\right)^{2} \int_{\frac{2 \pi}{\sqrt{N S_{0}}}}^{\frac{2 \pi}{d}} \int_{0}^{2 \pi} \frac{k T}{N S_{0}\left(\kappa_{B}|\boldsymbol{q}|^{4}+\alpha \beta\right)}|\boldsymbol{q}| d \theta d|\boldsymbol{q}| .
$$



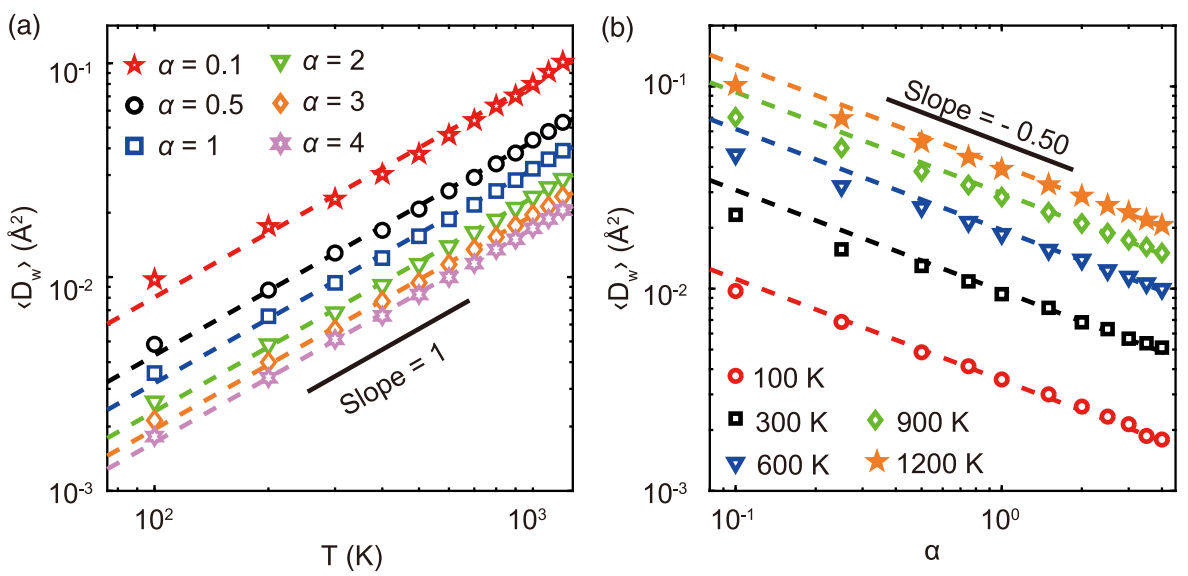

Fig. 4. The average amplitude of surface corrugations of SLG as a function of temperature and interaction strength. (a) $\left\langle D_{w}\right\rangle$ vs. temperature, (b) $\left\langle D_{w}\right\rangle$ vs. interaction strength. Here symbols represent the molecular dynamics simulation results and the dashed lines are the corresponding fitting curves. The slopes from MD simulations match well with the statistical mechanical analysis in Eq. (9).

The above integral gives rise to

$$
\left\langle D_{w}\right\rangle=\frac{k T}{4 \pi \sqrt{\kappa_{B} \alpha \beta}} \arctan \left(4 \pi^{2} \frac{\sqrt{w_{e q}^{2} S_{0}}}{d^{2}} \sqrt{\frac{\kappa_{B}}{72 \alpha \varepsilon}}\right) .
$$

Noticing $\frac{\sqrt{w_{e q}^{2} s_{0}}}{d^{2}} \sim 1$ and $\kappa_{B} \sim 100 \varepsilon$ (Xu and Buehler, 2010; Shi et al., 2012; Wei et al., 2013), hence the term $\arctan (\cdot)$ in Eq. (8c) is about $\pi / 2$ for a wide range of $\alpha$. In view of this, we have the following simplified form for $\left\langle D_{w}\right\rangle$,

$$
\left\langle D_{w}\right\rangle \approx \frac{k T}{8 \sqrt{\kappa_{B} \alpha \beta}} .
$$

When $\alpha \rightarrow 0$, we have $\left\langle D_{w}\right\rangle \rightarrow \infty$, which means that under the harmonic approximation large size graphene is unstable. This is consistent with the finding in literature (Mermin, 1968).

Below is the analysis of thermally induced ripples from our MD simulations. Fig. 4(a) shows the dependence of the variance of the height distribution on temperature. Eq. (9) predicts that $\left\langle D_{w}\right\rangle$ is proportional to temperature as long as the sample is sufficiently large $(\sqrt{N} \gg 1)$. It is noted here that the expression in Eq. (9) ignores the height fluctuation due to the formation of Moiré patterns seen in Fig. 2, which was taken into account by adding an offset term $\delta$ to $\left\langle D_{w}\right\rangle_{\text {sum }}$ from our simulations, i.e., $\left\langle D_{w}\right\rangle_{\text {sum }}=\left\langle D_{w}\right\rangle+\delta$. The offset term is independent of temperature but relies on the interaction strength and the relative crystallographic orientations of the graphene membrane and the substrate (Deng et al., 2017). Our simulation results over a wide range of temperatures from $100 \mathrm{~K}$ to $1200 \mathrm{~K}$ agree well with the statistical mechanics predictions using Eq. (9). Fig. 4(b) also shows that $\left\langle D_{w}\right\rangle$ is proportional to $\alpha^{-1 / 2}$. The slight deviation at high temperature is due to the dependence of $\delta$ on $\alpha$, which is neglected in the statistical mechanics model derived above.

\section{The relationship between surface corrugations and adhesion}

The appearance of surface corrugations along with temperature is able to modulate the adhesion property of membranes with a substrate. To be specific, the height fluctuation of the single layer graphene sheet due to thermal undulations in turn affects the interaction of graphene with the substrate. The presence of SLG membrane serving as a buffer layer, as it does in most of the practical applications, may enhance the interaction range of the graphene-substrate system with an external object. To understand the adhesion range between another body and the graphene-buffered substrate, a series of MD simulations were performed while the detailed procedure is stated in Section 2.1. The schematic diagram is shown in Fig. 5(a). For a model demonstration, we considered another block of copper with $\mathrm{Cu}$ (111) as its surface to be in contact with the graphene-buffered $\mathrm{Cu}$ substrate. The block was placed at a distance far beyond the adhesion range between its (111) surface and the graphene layer in the graphene-buffered substrate. The gap between the two $\mathrm{Cu}$ blocks was denoted as $\Delta$. By moving the two parts closer to each other so that they may interact in the statistical sense, we then employed a distance-controlled loading method to obtain the stress - separation relationship between the Cu block and graphenebuffered $\mathrm{Cu}$ substrate. At each $\Delta$, we relaxed the system in an NVT ensemble till the relative potential energy fluctuation is under $0.003 \%$. The cohesive stress $\sigma$ was then obtained by averaging the total interaction force between the SLG and the copper substrate.

Through the same loading steps, we obtained the stress and separation curves, i.e., $\sigma-\Delta$ relationship between the Cu block and the graphene-buffered $\mathrm{Cu}$ substrate. In Fig. 5(b), the stress and separation curves for a small interaction strength 
(a)
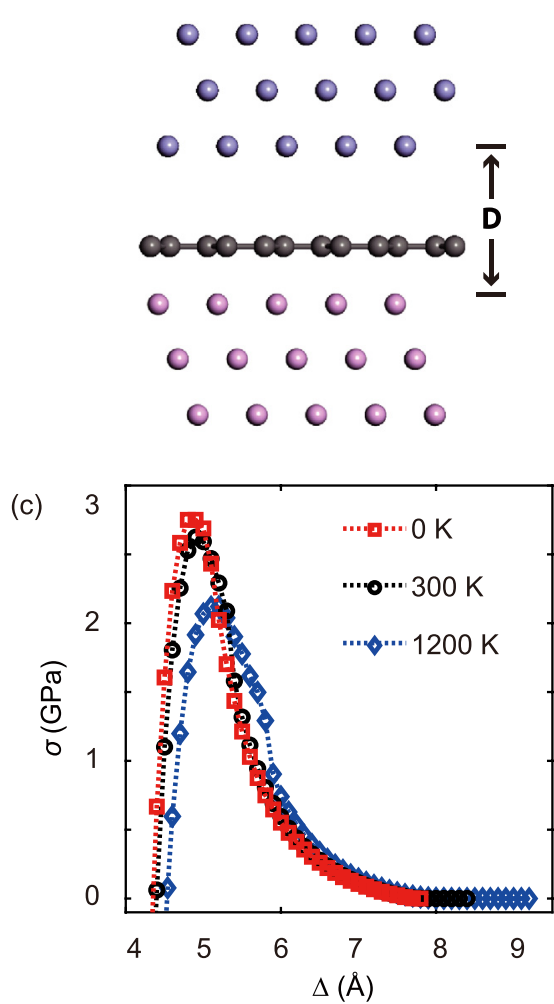
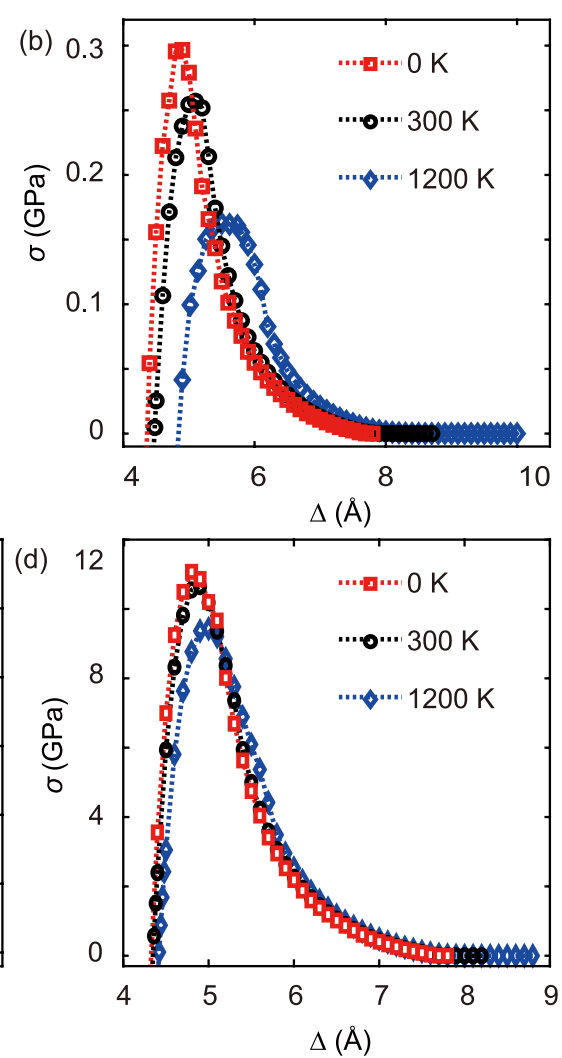

Fig. 5. Stress-separation curves for SLG-substrate systems. (a) Illustration of a simplified model for MD simulations and theoretical analysis of graphenebuffered interface. (b)-(d) The $\sigma-\Delta$ curves for $\alpha=0.1,1$ and 4 , respectively. The results are shown for three temperatures, at $T=0 \mathrm{~K}, 300 \mathrm{~K}$ and $1200 \mathrm{~K}$.

$\alpha=0.1$ is shown, and the temperature dependence is also explored by performing simulations at various temperatures $T=0 \mathrm{~K}, 300 \mathrm{~K}$ and $1200 \mathrm{~K}$, respectively. In Fig. 5(b)-(d), a significant temperature dependence of the maximum stress $\sigma_{m}$ is observed: $\sigma_{m}$ drops when temperature rises

Such strong dependence of $\sigma_{m}$ on temperature may be captured from the statistical mechanics analysis (Wei, 2014). In the presence of statistical bonding and debonding across an interface, the form of average stress $\langle\sigma(\Delta)\rangle$ can be expressed using the distribution of potential interatomic bonding sites $\rho_{C}(w)$ which is a function of separation, and the probability of those potential sites to form effective bonding $b(w, t)$, which varies with time for non-equilibrium state, and the resistant force $-\frac{\partial E(\Delta-w)}{\partial w}$ supplied by those effectively bonded sites. Here, $w$ itself is a statistical number, $\Delta-w$ is the gap between carbon atoms in graphene and the top $\mathrm{Cu}(111)$ block, and $E(\Delta-w)$ stands for their interaction energy. The averaged stress is written as

$$
\langle\sigma(\Delta)\rangle=\int_{0}^{\Delta} \rho_{C}(w) b(w, t) \frac{\partial E(\Delta-w)}{\partial w} d w
$$

where the product of $\rho_{C}(w) b(w, t)$ gives the number of effective bonding sites per unit area. As discussed in previous work (Shi et al., 2008; Qian et al., 2009; Wei, 2014), the reaction rate could be specific and is a function of time. In that sense, we may assign $\rho_{C}(w)$ as the density of specific bonding sites, and associate $b(w, t)$ with the separation distance and time-dependent bonding probability. For simplicity but without loss of physics, we set $b=1$ to explore the influence of temperature on morphology. In recognition of Fig. 2(c) and (d), the bonding density $\rho_{C}(w) b(w)$ may be assumed to follow the Gaussian distribution. Thus, we have the averaged stress $\langle\sigma(\Delta)\rangle$ :

$$
\langle\sigma(\Delta)\rangle=\int \frac{1}{\sqrt{2 \pi\left\langle D_{w}\right\rangle}} e^{-\frac{\left(w-w_{e q}\right)^{2}}{2\left(D_{w}\right)}} \frac{\partial E(\Delta-w)}{\partial w} d w .
$$

When the interaction $E(\Delta-w)$ is depicted by the L-J pair potential for $\mathrm{Cu}$ and $\mathrm{C}$ atoms, the explicit form of $\langle\sigma(\Delta)\rangle$ is not available due to the complexity of the integrand. Consequently, we perform the Taylor expansion on $\frac{\partial E(\Delta-w)}{\partial w}$ to quantify the dependence of $\sigma_{m}$ on $\alpha$ and $T$. The expansion is carried out at the point where the maximum stress appears for a single 

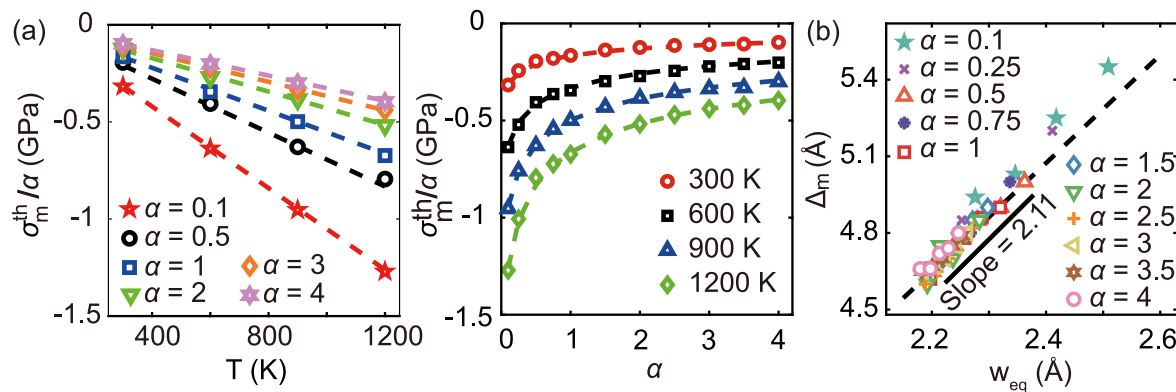

Fig. 6. The analysis for the adhesion between single layer graphene membrane and a copper substrate. (a) The maximum separation strength as a function of temperature (left) and as a function of interaction strength (right). Here the symbols represent the results from MD simulations and the dashed lines are predictions using Eq. (12c). (b) The variation of the interaction distance as a function of temperature and interaction strength. The symbols represent the MD simulation results and the dashed lines are the corresponding fitting lines.

C-Cu pair with L-J interaction, i.e., at $w_{m}=\sqrt[6]{\frac{26}{7}} r_{0}$. Keeping the first two terms, we have

$$
\frac{\partial E(\Delta-w)}{\partial w}=\frac{504}{169} \frac{\alpha \varepsilon}{S_{0} w_{m}}-\frac{1764}{13} \frac{\alpha \varepsilon}{S_{0} w_{m}^{3}}\left[(w-\Delta)-w_{m}\right]^{2},
$$

and the average stress $\langle\sigma(\Delta)\rangle$ becomes

$$
\langle\sigma(\Delta)\rangle \approx \frac{504}{169} \frac{\alpha \varepsilon}{S_{0} w_{m}}-\frac{1764}{13} \frac{\alpha \varepsilon}{S_{0} w_{m}^{3}}\left[\left(\Delta-w_{e q}-w_{m}\right)^{2}+\left\langle D_{w}\right\rangle\right] .
$$

Now the maximum stress $\sigma_{m}$ in the $\langle\sigma\rangle-\Delta$ curve appears at

$$
\Delta_{m}=w_{e q}+w_{m}=2.11 w_{e q},
$$

with

$$
\sigma_{m}=\frac{504}{169} \frac{\alpha \varepsilon}{S_{0} w_{m}}-\frac{1764}{13} \frac{\alpha \varepsilon}{S_{0} w_{m}^{3}}\left\langle D_{w}\right\rangle .
$$

As shown in Eq. (12b), the dependence of $\sigma_{m}$ on $T$ and $\alpha \varepsilon$ could be rationalized as follows. The strength of materials is normally composed of two contributions, i.e., $\sigma_{m}=\sigma_{m}^{a}+\sigma_{m}^{\text {th }}$, a thermally dependent term $\sigma_{m}^{\text {th }}$ and an athermal term $\sigma_{m}^{a}$. Here $\sigma_{m}^{a}=\frac{504}{169} \frac{\alpha \varepsilon}{S_{0} w_{m}}$ is the maximum stress between a flat single layer graphene membrane and the $\mathrm{Cu}$ (111) block and is essentially independent of temperature, which is determined by the interaction strength and is slightly influenced by the Moiré pattern. However, $\sigma_{m}^{\text {th }}=-\frac{1764}{13} \frac{\alpha \varepsilon}{S_{0} w_{m}^{3}}\left\langle D_{w}\right\rangle$, is a function of both $T$ and $\alpha$, as shown Eq. (9). Numerical method is then used to obtain the exact stress-separation curves and the corresponding strength $\sigma_{m}$. The results could be better fitted by modifying Eq. (12b) in the following form as the derivation given in Eq. (12b) is an approximation,

$$
\frac{\sigma_{m}^{t h}}{\alpha}=-\eta \frac{\varepsilon}{S_{0} w_{m}^{3}} \frac{k T}{8 \sqrt{\kappa_{B} \alpha \beta}+\xi} .
$$

Assuming $\eta=253.27$ and $\xi=1.16 \mathrm{eV} / \AA^{2}$, this formula matches well with our MD simulation results of $\sigma_{m}^{\text {th }}$ vs. $T$ and $\sigma_{m}^{\text {th }}$ vs. $\alpha$, as shown in Fig. 6(a). Fig. 5(b)-(d) show that the position $\Delta_{m}$, where $\sigma_{m}$ is at its maximum, is also influenced by $T$ and $\alpha$. Both $w_{e q}$ and $\left\langle D_{w}\right\rangle$ represent the amplitude of the surface corrugations and they share a similar correlation with $T$ and $\alpha$. As seen in Eq. (12a), $\Delta_{m}$ is proportional to the equilibrium position $w_{e q}$. Fig. 6(b) shows the dependence of $\Delta_{m}$ on $w_{e q}$ from MD simulations, which is in excellent agreement with the statistical mechanics predictions using Eq. (12a).

The results in Fig. 5 demonstrate that the adhesion range between the two rigid bodies is extended when a single layer graphene membrane is used as a buffer layer. The rippling of the single layer graphene membrane increases as temperature increases and when graphene-substrate interaction becomes weaker. To find the exact adhesion range, i.e., the separation distance when the attractive stress approaches zero, we pay more attention to the region of the curves when normal traction becomes negligible. As the occurrence of interaction is random, the carbon atoms are likely to distribute within a distance of $3 \sqrt{\left\langle D_{w}\right\rangle}$ from their equilibrium positions by noticing the characteristics of the Gaussian distribution. Eq. (9) illustrates the enhancement of the adhesion range as temperature rises or the interacting strength becomes weaker. At the low temperature limit, thermally induced rippling is suppressed and the interaction becomes negligible beyond a cut-off distance $\Delta$ as described by the L-J potential for C-Cu pairs, i.e., the adhesion range is $\Delta=7.8 \AA$. When $\alpha$ is small, i.e., $\alpha=0.1$, the adhesion range could be as far as $1 \mathrm{~nm}$. It should be noted that the above analysis considers only the thermal undulation of carbon atoms in SLG. In the presence of residual stress, defects in graphene or ridges in the substrate, SLG can form nanometer-height wrinkles (Deng et al., 2017), which could significantly change the adhesion nature. 
(a)

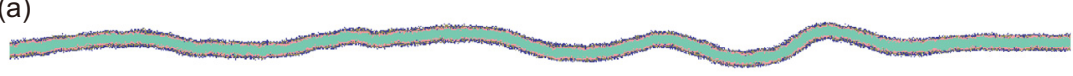

(b)

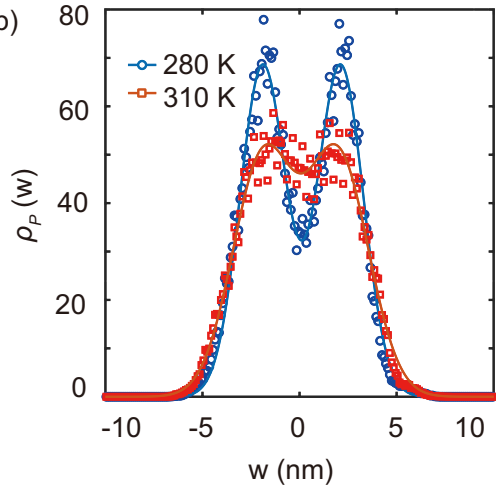

(c)

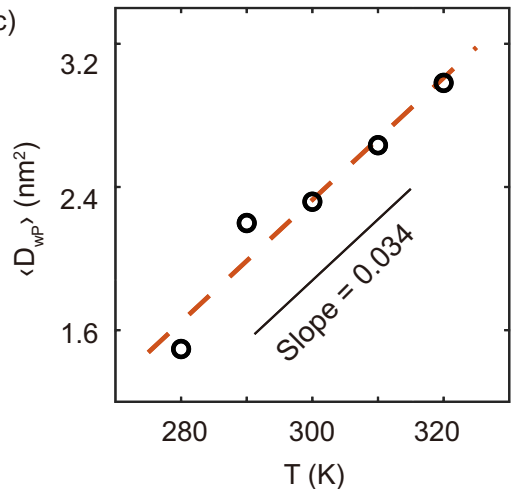

Fig. 7. Morphology of POPC bilayers at various temperatures. (a) A snapshot of a free-standing POPC bilayer at $310 \mathrm{~K}$. (b) The distribution of PO4 particles at $280 \mathrm{~K}$ and $310 \mathrm{~K}$. The double peak shown here is due to the top and bottom molecules in the POPC bilayer, which gives rise to the bi-Gaussian distribution seen here. The distance of the two peaks stands for the average thickness of the bilayer. (c) The fluctuation amplitude $\left\langle D_{w P}\right\rangle$ of free-standing bilayers at various temperatures.

\section{The enhanced adhesion range of lipid bilayers}

Another typical and important membrane adhesion scenario is the interaction of lipid bilayers with substrates. Following a similar approach, we demonstrate using results from molecular dynamics simulations and theoretical analysis that our statistical mechanics model could be used to capture the adhesion of lipid bilayers on a substrate.

\subsection{Lipid bilayer - substrate interaction}

Considering that the bending rigidity of single layer graphene is close to that of cell lipid bilayers, the enhanced adhesion range of graphene-buffered substrate due to thermal undulation may mechanically justify the similar behavior in the biological systems where adhesion controls biological functionality. Indeed, membrane rippling, either thermally induced or mechanically activated, is an effective factor mediating cell cohesion and drug delivery, as demonstrated experimentally (Nel et al., 2009; Sarfati and Dufresne, 2016) and through theoretical studies (Podgornik and Parsegian, 1992; Gao et al., 2005; Lin et al., 2008). Surface undulation increases the chance of bonding between the proteins in neighboring cell membranes (Fenz et al., 2017) as the amplitudes of the undulations could be in the nanometer scale.

MD simulations were performed to model the adhesion behavior of 1-palmitoyl-2-oleoyl-sn-glycero-3-phosphocholine (POPC) bilayers with a substrate. The substrate is rigid and the motion of all particles are constrained. A rectangular lipidbilayer with the size of $4000 \mathrm{~nm} \times 10 \mathrm{~nm}$ was used. The coarse-grained model developed by Marrink et al. (Marrink et al., 2004, 2007; Arnarez et al., 2015) was adopted. We neglected the influence from water molecules on the thermal fluctuation and used the Dry Martini Force Field (Arnarez et al., 2015) to describe the interactions within the lipid bilayer. The cut-off of the non-bonded interaction was set to be $1.2 \mathrm{~nm}$ (Wang et al., 2013; Arnarez et al., 2015). The simulations for the lipidbilayers were performed by using GROMACS (Pronk et al., 2013) and were visualized with VMD (Humphrey et al., 1996).

To mimic real biological environment, a free-standing lipid bilayer was first relaxed in NPT ensembles at temperature range of $280 \sim 320 \mathrm{~K}$ and at a pressure of 1 bar. Periodic boundary was employed in the $x-y$ plane. The solvent-free simulations were performed by the stochastic dynamics (SD) integrator and the pressure was controlled by Berendsen barostat with semi-isotropic pressure coupling. The time used in the simulation could be converted to the effective time conveniently by multiplying a speed up factor of 4 .

For the substrates of the supported bilayers, we adopted a model from literature (Xing and Faller, 2008, 2009). The corresponding CG particles with a radius of $0.47 \mathrm{~nm}$ were employed. The particles were fixed in a square array with a spacing of $0.3 \mathrm{~nm}$. The interaction between the lipid particles and the substrate is described by the L-J potential (Xing and Faller, 2008). All simulations regarding supported systems were performed in NVT ensemble.

\subsection{Temperature-dependent adhesion of lipid bilayers}

As shown in Fig. 7(a), surface fluctuation appears in the lipid bilayer after a relaxation of $0.1 \mu$ s. At each temperature, we generated multiple configurations for statistical analysis. As a lipid-bilayer has a finite thickness, the positions of PO4 beads in the top and bottom surface of the bilayer were taken to represent its morphology. The distribution of PO4 beads at $280 \mathrm{~K}$ 

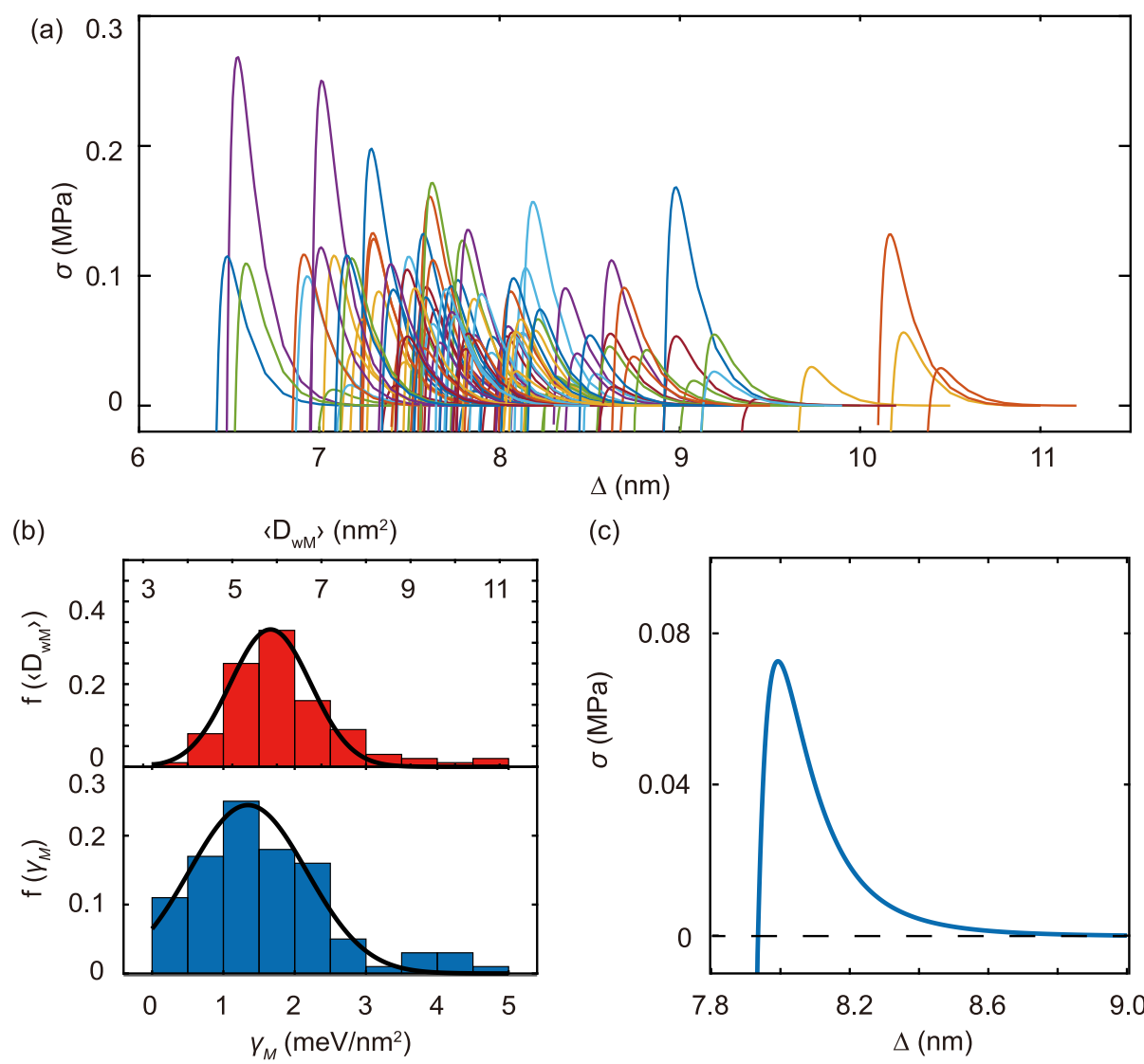

(c)

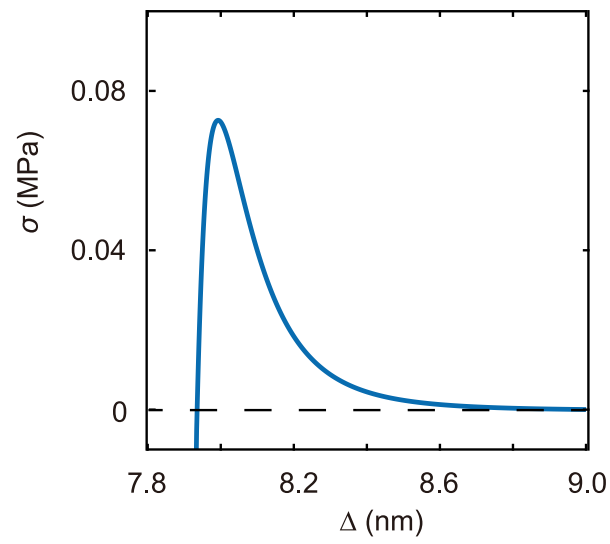

Fig. 8. Statistic adhesion of lipid bilayers on a substrate. (a) Fluctuation induced scattering of stress-separation response from 100 configurations of the same POPC bilayer. (b) Distribution of $\left\langle D_{w M}\right\rangle$ and $\gamma_{M}$ which characterize the interaction range and interaction strength of the bilayer. (c) The averaged $\Delta-\sigma$ curve of the bilayer.

and $310 \mathrm{~K}$ are shown in Fig. 7(b). The out-of-plane displacement $w$ of PO4 beads follows a bimodal Gaussian distribution

$$
\rho_{P}(w)=\frac{1}{2 \sqrt{2 \pi\left\langle D_{w P}\right\rangle}}\left\{\exp \left[-\frac{\left(w-\bar{w}_{1}\right)^{2}}{2\left\langle D_{w P}\right\rangle}\right]+\exp \left[-\frac{\left(w-\bar{w}_{2}\right)^{2}}{2\left\langle D_{w P}\right\rangle}\right]\right\}
$$

where the two peaks $\bar{w}_{1}=-2.1 \pm 0.03 \mathrm{~nm}$ and $\bar{w}_{2}=2.1 \pm 0.03 \mathrm{~nm}$, respectively, indicating the nominal thickness of the bilayer is $4.22 \mathrm{~nm}$, consistent with the previous numerical calculations conducted by Arnarez et al. (2015). $\left\langle D_{w P}\right\rangle$ stands for the mean-square fluctuation amplitude of the bilayer. It follows a linear relationship with temperature, as predicted by Eq. (9) and seen in Fig. 7(c).

To illustrate how such thermally induced fluctuation in lipid bilayer may influence adhesion, a similar simulation strategy, as shown above for SLG, was followed. Relaxed POPC ribbons was initially far away from a weak hydrophilic substrate. Defining a separation $\Delta$ which is the distance between the substrate surface and the center of mass of the ribbon, the interaction force between the lipid bilayer and the substrate was calculated as a function of $\Delta$. The configurations are generated at $310 \mathrm{~K}$ to study its adhesion properties in a practical temperature environment. More than 100 independent configurations of the POPC lipid bilayer were acquired after relaxation for $100 \mathrm{~ns}$. Fig. 8(a) shows a total of 100 typical $\Delta-\sigma$ curves from different configurations. The scattering of the adhesion behavior originates from the fluctuation of the lipid bilayer, which has to be understood using statistical mechanics. Each $\Delta-\sigma$ curve is characterized by its zero-stress point at the distance of $\Delta_{0}$, its maximum stress point $\Delta_{\max }$, and the point of convergence $\Delta_{\text {end }}$ beyond which the interaction is negligible.

To unify those distinct $\Delta-\sigma$ curves of initially different lipid-bilayer configurations, we adopted the stress - separation constitutive model from statistical analysis (Wei, 2014). Eq. (10a) may be rewritten in the form of

$$
\langle\sigma(\Delta)\rangle=\int_{0}^{\Delta} \rho_{M}(\Delta, \mathrm{w}) b(w) \frac{\partial E(w)}{\partial w} d w .
$$

The effective density of bonding sites follows the Gaussian distribution

$$
\rho_{M}(\Delta, w) b(w, t)=\frac{1}{\sqrt{2 \pi\left\langle D_{w M}\right\rangle}} e^{-\frac{(w-\Delta)^{2}}{2\left\langle D_{w M}\right\rangle}}
$$




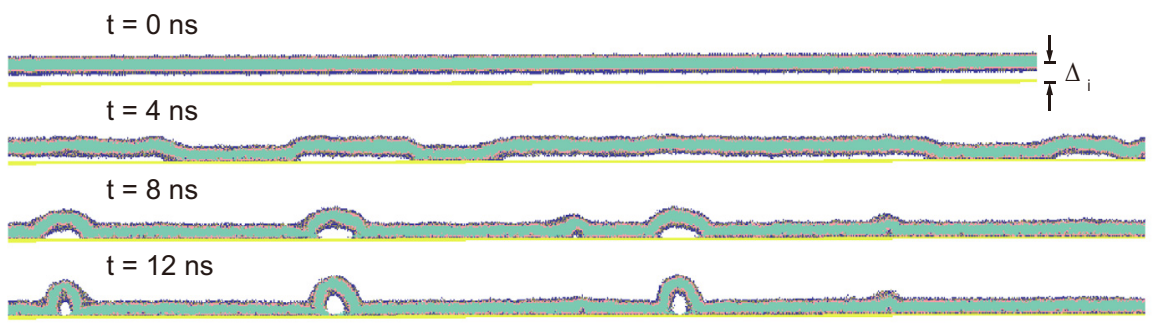

Fig. 9. The dynamics of the POPC bilayer adhering to the substrate at $310 \mathrm{~K}$ and with an initial separation of $4.3 \mathrm{~nm}$.

The potential $E(w)$ is described by the L-J form

$$
E(w)=4 \gamma_{M}\left[\left(\frac{w_{0}}{w}\right)^{12}-\left(\frac{w_{0}}{w}\right)^{6}\right]
$$

with $\gamma_{M}$ being the potential energy normalized by the area of a bonding site, and $w_{0}=0.47 \mathrm{~nm}$ in the simulation (Marrink, et al., 2004; Marrink, et al., 2007; Arnarez et al., 2015). To represent the $\Delta-\sigma$ curves in Fig. 8(a), we have different $\left\langle D_{w M}\right\rangle$ and $\gamma_{M}$ for each curve, i.e., the bonding energy and bonding distance and they both are statistic in nature. Their respective distributions $f\left(\left\langle D_{w M}\right\rangle\right)$ and $f\left(\gamma_{M}\right)$ are shown in Fig. 8(b). The averaged $\Delta-\sigma$ curves in Fig. 8(a) could then be abstracted by substituting the mean $\gamma_{M}$ and mean $\left\langle D_{w M}\right\rangle$ in Fig. 8(b) into Eq. (14a), which is shown in Fig. 8(c).

Thermal fluctuation mediated adhesion could be further demonstrated by examining the dynamics of adhesion. An initially flat bilayer was transferred to substrate with an initial separation of $\Delta_{i}$. We first put the POPC ribbon $4.30 \mathrm{~nm}$ away from the substrate, note this $\Delta_{i}$ is far beyond the interaction range of $3.31 \mathrm{~nm}$. Thermal fluctuation induced ripples facilitate the approaching of the lipid bilayer membrane to the substrate, leading to the adherence of both, as seen from the dynamic morphology at different time in Fig. 9.

\section{Conclusion}

To summarize, we report in this work the linear temperature-dependence of adhesion strength in soft membrane interfaces including graphene and lipid bilayers. Such a strong temperature effect on adhesion strength has never been predicted by the classical mechanics theories before. Via a combination of statistical mechanics analysis and MD simulations, we demonstrated that the high sensitivity of adhesion on temperature results from thermally induced ripples. An enhancement of the adhesion range and a linear variation of the adhesion strength could be observed as temperature rises, which facilitates long-range adhesion between soft membranes like graphene sheets and lipid-bilayers with a substrate. Such surprising findings may pave the way to understand temperature-sensitive kinetics in many biological systems where the interaction of cell membranes with extra-cellular environments is pivotal.

\section{Acknowledgments}

Y.W. acknowledges support from the National Natural Science Foundation of China (Grant No. 11425211), the Strategic Priority Research Program of the Chinese Academy of Sciences (XDB22020200), and CAS Center for Excellence in Complex System Mechanics. The simulations are conducted at the Supercomputing Center of CAS.

\section{References}

Abbena, E., Salamon, S., Gray, A., 2006. Modern Differential Geometry of Curves and Surfaces with Mathematica. CRC Press.

Ahmadpoor, F., Wang, P., Huang, R., Sharma, P., 2017. Thermal fluctuations and effective bending stiffness of elastic thin sheets and graphene: a nonlinear analysis. J. Mech. Phys. Solids 107, 294-319.

Arnarez, C., Uusitalo, J.J., Masman, M.F., Ingolfsson, H.I., de Jong, D.H., Melo, M.N., Periole, X., de Vries, A.H., Marrink, S.J., 2015. Dry martini, a coarse-grained force field for lipid membrane simblations with implicit solvent. J. Chem. Theory Comput. 11 (1), 260-275.

Castellana, E.T., Cremer, P.S., 2006. Solid supported lipid bilayers: from biophysical studies to sensor design. Surf. Sci. Rep. 61 (10), $429-444$.

Deng, B., Pang, Z.Q., Chen, S.L., Li, X., Meng, C.X., Li, J.Y., Liu, M.X., Wu, J.X., Qi, Y., Dang, W.H., Yang, H., Zhang, Y.F., Zhang, J., Kang, N., Xu, H.Q., Fu, Q., Qiu, X.H., Gao, P., Wei, Y.J., Liu, Z.F., Peng, H.L., 2017. Wrinkle-free single-crystal graphene wafer grown on strain-engineered substrates. ACS Nano 11 (12), 12337-12345.

Deng, S.K., Berry, V., 2016. Wrinkled, rippled and crumpled graphene: an overview of formation mechanism, electronic properties, and applications. Mater. Today 19 (4), 197-212.

Devaux, P.F., 1991. Static and dynamic lipid asymmetry in cell-membranes. Biochemistry 30 (5), 1163-1173.

Dobereiner, H.G., 1999. Quantifying membrane asymmetry. Biophys. J. 76 (4), 1723-1724.

Dobereiner, H.G., Selchow, O., Lipowsky, R., 1999. Spontaneous curvature of fluid vesicles induced by trans-bilayer sugar asymmetry. Eur. Biophys. J. Biophys. Lett. 28 (2), 174-178.

Drotlef, D.M., Amjadi, M., Yunusa, M., Sitti, M., 2017. Bioinspired composite microfibers for skin adhesion and signal amplification of wearable sensors. Adv. Mater. 29 (28), 1701353.

Fasolino, A., Los, J.H., Katsnelson, M.I., 2007. Intrinsic ripples in graphene. Nat. Mater. 6 (11), 858-861. 
Fenz, S.F., Bihr, T., Schmidt, D., Merkel, R., Seifert, U., Sengupta, K., Smith, A.S., 2017. Membrane fluctuations mediate lateral interaction between cadherin bonds. Nat. Phys. 13 (9), 906-913.

Foiles, S.M., Baskes, M.I., Daw, M.S., 1986. Embedded-atom-method functions for the Fcc metals Cu, Ag, Au, Ni, Pd, Pt, and their alloys. Phys. Rev. B 33 (12), 7983-7991.

Freund, L.B., 2013. Entropic pressure between biomembranes in a periodic stack due to thermal fluctuations. Proc. Natl. Acad. Sci. USA 110 (6), 2047-2051.

Gao, H.J., Shi, W.D., Freund, L.B., 2005. Mechanics of receptor-mediated endocytosis. Proc. Natl. Acad. Sci. USA 102 (27), $9469-9474$.

Gao, L., Guest, J.R., Guisinger, N.P., 2010. Epitaxial graphene on Cu(111). Nano Lett. 10 (9), 3512-3516.

Gao, W., Huang, R., 2014. Thermomechanics of monolayer graphene: rippling, thermal expansion and elasticity. J. Mech. Phys. Solids 66, 42-58.

Geringer, V., Liebmann, M., Echtermeyer, T., Runte, S., Schmidt, M., Ruckamp, R., Lemme, M.C., Morgenstern, M., 2009. Intrinsic and extrinsic corrugation of monolayer graphene deposited on $\mathrm{SiO}_{2}$. Phys. Rev. Lett. 102 (7), 076102.

Gouliaev, N., Nagle, J.F., 1998. Simulations of interacting membranes in the soft confinement regime. Phys. Rev. Lett. 81 (12), $2610-2613$.

Helfrich, W., 1973. Elastic properties of lipid bilayers - Theory and possible experiments. Zeitschrift Fur Naturforschung C-a J. Biosci. C 28 (11-1), 693-703.

Helfrich, W., 1978. Steric interaction of fluid membranes in multilayer systems. Zeitschrift Fur Naturforschung Section a-a J. Phys. Sci. 33 (3), $305-315$.

Humphrey, W., Dalke, A., Schulten, K., 1996. VMD: visual molecular dynamics. J. Mol. Graph. Model. 14 (1), 33-38.

Ishigami, M., Chen, J.H., Cullen, W.G., Fuhrer, M.S., Williams, E.D., 2007. Atomic structure of graphene on SiO 2 . Nano Lett. 7 (6), $1643-1648$.

Katsaras, J., Gutberlet, T., 2000. Lipid Bilayers Structure and Interactions. Springer, Berlin Heidelberg.

Lee, C., Li, Q.Y., Kalb, W., Liu, X.Z., Berger, H., Carpick, R.W., Hone, J., 2010. Frictional characteristics of atomically thin sheets. Science 328 (5974), 76-80.

Li, L., Song, F., 2016. Entropic force between biomembranes. Acta Mechanica Sinica 32 (5), 970-975.

Li, L., Song, F., 2018. Undulation force between membranes. Adv. Mech. 48, 438-458.

Li, Y.F., Yuan, H.Y., von dem Bussche, A., Creighton, M., Hurt, R.H., Kane, A.B., Gao, H.J., 2013. Graphene microsheets enter cells through spontaneous membrane penetration at edge asperities and corner sites. Proc. Natl. Acad. Sci. USA. 110 (30), 12295-12300.

Lin, Y., Inamdar, M., Freund, L.B., 2008. The competition between Brownian motion and adhesion in soft materials. J. Mech. Phys. Solids 56 (1), 241-250.

Lipowsky, R., 1991. The conformation of membranes. Nature 349 (6309), 475-481.

Marrink, S.J., de Vries, A.H., Mark, A.E., 2004. Coarse grained model for semiquantitative lipid simulations. J. Phys. Chem. B 108 (2), $750-760$.

Marrink, S.J., Risselada, H.J., Yefimov, S., Tieleman, D.P., de Vries, A.H., 2007. The martini force field: coarse grained model for biomolecular simulations. J. Phys. Chem. B 111 (27), 7812-7824.

Mermin, N.D., 1968. Crystalline order in 2 dimensions. Phys. Rev. 176 (1), 250-254.

Nel, A.E., Madler, L., Velegol, D., Xia, T., Hoek, E.M.V., Somasundaran, P., Klaessig, F., Castranova, V., Thompson, M., 2009. Understanding biophysicochemical interactions at the nano-bio interface. Nat. Mater. 8 (7), 543-557.

Nelson, D.R., Peliti, L., 1987. Fluctuations in membranes with crystalline and hexatic order. J. De Physique 48 (7), $1085-1092$.

Nelson, D.R., Piran, T., Weinberg, S., 2004. Statistical Mechanics of Membranes and Surfaces, 2nd editon. World Scientific Publishing Co. Pte. Ltd.

Petrache, H.I., Gouliaev, N., Tristram-Nagle, S., Zhang, R.T., Suter, R.M., Nagle, J.F., 1998. Interbilayer interactions from high-resolution x-ray scattering. Phys. Rev. E 57 (6), 7014-7024.

Plimpton, S., 1995. Fast parallel algorithms for short-range molecular-dynamics. J. Comput. Phys. 117 (1), 1-19.

Podgornik, R., Parsegian, V.A., 1992. Thermal mechanical fluctuations of fluid membranes in confined geometries - the case of soft confinement. Langmuir 8 (2), 557-562.

Pronk, S., Pall, S., Schulz, R., Larsson, P., Bjelkmar, P., Apostolov, R., Shirts, M.R., Smith, J.C., Kasson, P.M., van der Spoel, D., Hess, B., Lindahl, E., 2013. GROMACS 4.5: a high-throughput and highly parallel open source molecular simulation toolkit. Bioinformatics 29 (7), $845-854$.

Qian, J., Wang, J.Z., Lin, Y., Gao, H.J., 2009. Lifetime and strength of periodic bond clusters between elastic media under inclined loading. Biophys. J. 97 (9), $2438-2445$.

Sarfati, R., Dufresne, E.R., 2016. Long-range attraction of particles adhered to lipid vesicles. Phys. Rev. E 94 (1).

Seifert, U., Lipowsky, R., 1990. Adhesion of vesicles. Phys. Rev. A 42 (8), 4768-4771.

Seung, H.S., Nelson, D.R., 1988. Defects in flexible membranes with crystalline order. Phys. Rev. A 38 (2), $1005-1018$.

Shenoy, V.B., Reddy, C.D., Ramasubramaniam, A., Zhang, Y.W., 2008. Edge-Stress-Induced warping of graphene sheets and nanoribbons. Phys. Rev. Lett. 101 (24), 245501

Shi, X.H., Yin, Q.F., Wei, Y.J., 2012. A theoretical analysis of the surface dependent binding, peeling and folding of graphene on single crystal copper. Carbon 50 (8), 3055-3063.

Shi, W., Wang, J.Z., Fan, X.J., Gao, H.J., 2008. Size and shape effects on diffusion and absorption of colloidal particles near a partially absorbing sphere: implications for uptake of nanoparticles in animal cells. Phys. Rev. E 78, 061914.

Stuart, S.J., Tutein, A.B., Harrison, J.A., 2000. A reactive potential for hydrocarbons with intermolecular interactions. J. Chem. Phys. 112 (14), $6472-6486$.

Stukowski, A., 2010. Visualization and analysis of atomistic simulation data with OVITO-the open visualization tool. Model. Simul. Mater. Sci. Eng. 18 (1), 015012 .

Swain, P.S., Andelman, D., 1999. The influence of substrate structure on membrane adhesion. Langmuir 15 (26), $8902-8914$.

Wang, J.L., Wei, Y.J., Shi, X.H., Gao, H.J., 2013. Cellular entry of graphene nanosheets: the role of thickness, oxidation and surface adsorption. RSC Adv. 3 (36), 15776-15782

Wang, P., Gao, W., Huang, R., 2016. Entropic effects of thermal rippling on van der Waals interactions between monolayer graphene and a rigid substrate. J. Appl. Phys. 119 (7).

Wei, Y.J., 2014. A stochastic description on the traction-separation law of an interface with non-covalent bonding. J. Mech. Phys. Solids 70, $227-241$.

Wei, Y.J., Wang, B.L., Wu, J.T., Yang, R.G., Dunn, M.L., 2013. Bending rigidity and Gaussian bending stiffness of single-layered graphene. Nano Lett. 13 (1), $26-30$.

Wei, Y.J., Wu, J.T., Yin, H.Q., Shi, X.H., Yang, R.G., Dresselhaus, M., 2012. The nature of strength enhancement and weakening by pentagon-heptagon defects in graphene. Nat. Mater. 11 (9), 759-763.

Wei, Y.J., Yang, R.G., 2019. Nanomechanics of graphene. Natl. Sci. Rev. 6 (2), 324-348.

Weiner, J.H., 2002. Statistical Mechanics of Elasticity, 2nd edition ed. Dover Publications, Mineola, New York.

Xing, C.Y., Faller, R., 2008. Interactions of lipid bilayers with supports: a coarse-grained molecular simulation study. J. Phys. Chem. B 112 (23), $7086-7094$.

Xing, C.Y., Faller, R., 2009. Coarse-grained simulations of supported and unsupported lipid monolayers. Soft Matter 5 (22), $4526-4530$.

Xu, Z.P., Buehler, M.J., 2010. Interface structure and mechanics between graphene and metal substrates: a first-principles study. J. Phys.-Condens. Matter 22 (48), 485301.

Yang, C.H., Suo, Z.G., 2018. Hydrogel ionotronics. Nat. Rev. Mater. 3 (6), 125-142.

Yu, Z.Z., Cheng, H.Y., 2018. Tunable adhesion for bio-integrated devices. Micromachines 9 (10).

Zang, J.F., Ryu, S., Pugno, N., Wang, Q.M., Tu, Q., Buehler, M.J., Zhao, X.H., 2013. Multifunctionality and control of the crumpling and unfolding of large-area graphene. Nat. Mater. 12 (4), 321-325.

Zelisko, M., Ahmadpoor, F., Gao, H.J., Sharma, P., 2017. Determining the Gaussian modulus and edge properties of 2D materials: from graphene to lipid bilayers. Phys. Rev. Lett. 119 (6), 068002.

Zhao, G.K., Li, X.M., Huang, M.R., Zhen, Z., Zhong, Y.J., Chen, Q., Zhao, X.L., He, Y.J., Hu, R.R., Yang, T.T., Zhang, R.J., Li, C.L., Kong, J., Xu, J.B., Ruoff, R.S., Zhu, H.W., 2017. The physics and chemistry of graphene-on-surfaces. Chem. Soc. Rev. 46 (15), 4417-4449. 